\title{
Development and Path Planning of a Low Cost Hexapod Mobile Robot
}

\author{
Afonso da Fonseca Braga * Exuperry Barros Costa * \\ Leonardo Rocha Olivi* Alexander Silva Barbosa* \\ * Electrical Engineering Department, Federal University of Juiz de \\ Fora, Juiz de Fora, Brazil (e-mails: afonso.braga@engenharia.ufjf.br, \\ exuperry.costa@ufjf.edu.br, leonardo.olivi@ufjf.edu.br, \\ alexander.silva@engenharia.ufjf.br).
}

\begin{abstract}
Computer Science and Robotics are becoming strong and consistent methods in teaching Science, Technology, Engineering, Arts and Mathematics (STEAM) in schools all over the world. In this sense, robots have been developed for schools and teaching, in several ways of configurations. There are many ways of locomotion for mobile robots, mainly based in wheels and mechanical legs. Unfortunately, there are few legged robots available in the market, and to the best of our knowledge there isn't a commercial model made in Brazil, which makes it more expensive to import. This paper proposes the development of a low cost fully functional hexapod, for educational purposes, describing its hardware and software, and also, showing control tests. The developed hexapod was conceived with 3D-printed legs and low-cost servomotors, that can be easily acquired and assembled. A transparent and effective API to control its movements, LEDs and other functionalities was developed to provide a quick learning curve for the user. The Hexapod's Kinematics chain and its motion strategy are discussed in depth, with a pseudoInverse Jacobian method to execute the legs Inverse Kinematic, allied with quintic polynomial function servo planner, successfully leading to a smooth movement to each hexapod's leg. The goal is to produce a low-cost, 3D printable robot for school education.
\end{abstract}

\section{Keywords: Hexapod, Mobile Robotics, Educational Robotics, Embedded Systems, Trajectory} Planning

\section{INTRODUCTION}

Autonomous Robotic Mechanical Systems are divided in two main areas: mobile robotics and manipulators. Manipulators are used to perform precise and fast tasks. Despite those qualities with a fixed basis they are not able to move around so easily. Mobile Robots are able to move through different environments using for example wheels or mechanical legs.

There are many ways of locomotion for mobile robots. Walking, jumping and crawling are examples of biological behaviours that inspired actual ways of locomotion, as can be seen in Hudson et al. (2018), Kasaei et al. (2017), Passault et al. (2016) and Koh and Cho (2013). Siegwart et al. (2011) compares the movement from different robot styles in a variety of environments. Robots with legs tend to be slower than wheeled robots for example. However, robots with legs can move better in rough terrains than wheeled robots.

Learning using Computer Science tools is becoming more attractive and accepted in schools all over the world. The work presented by Costelha and Neves (2018) shows a study to gather a robotic learning material for primary and secondary education (K-12 students). The work gathers several information about educational robotics, with robotic platforms, to be applied in Science, Technology, Engineering, Arts and Mathematics. Authors show how to use robotics in all grades for education purposes, showing the benefits of robotics in development of K-12.

In the paper presented by Passault et al. (2016), the authors present an open source robot designed for educational purposes. The robot is a four-legged 3D printed, with low cost, named Metabot. Its Inverse Kinematics is solved analytically for the Cartesian space. The programming environment is based in blocks, such as Lego Mindstorms or Anki's Cozmo. Authors proposed a competition among students and received good learning interaction. Silveira and Lacerda (2017) aims to develop a six-legged robot based on Arduino platform capable of obstacle avoidance, functional and economically viable.

A hexapod is a robot that has three pairs of legs, and can be more stable than a robot with fewer legs. Despite the stability that six legs can provide, the robot will consume more energy to power up those servomotors. Beyond those two facts, in case of a leg failure the hexapod will still be able to walk.

The present work aims to reports the development of a low cost fully functional hexapod, for educational purposes. To accomplish this task, its hardware and software are detailed, as the locomotion strategy and path planning of its six legs joints.

This paper is divided as follows. The development of hardware and software responsible for the vehicle control 


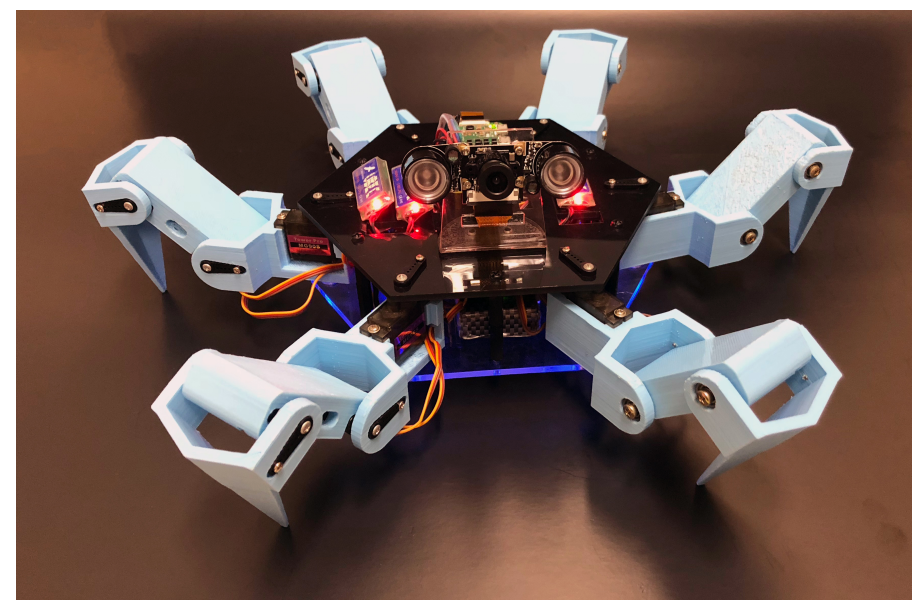

Figure 1. Hexapod Mobile Robot developed.

are described in Section 2. The robot Direct and Inverse Kinematics and its trajectory planning are discussed in Section 3. The locomotion strategies for hexapods are presented in Section 4. The obtained results are shown in Section 5, while Section 6 presents the main conclusions of this work.

\section{HEXAPOD DEVELOPMENT}

There are few hexapods available in the market. The final user in Brazil needs to choose between a low cost robot that does not have enough features and a high expensive model. Another fact is that imported models do not have enough documentations in Portuguese excluding the possibility of being used in every Brazilian school.

Focusing on educational robotics, this section presents the hardware and software design of a low cost hexapod. A prototype can be seen in Fig. 1.

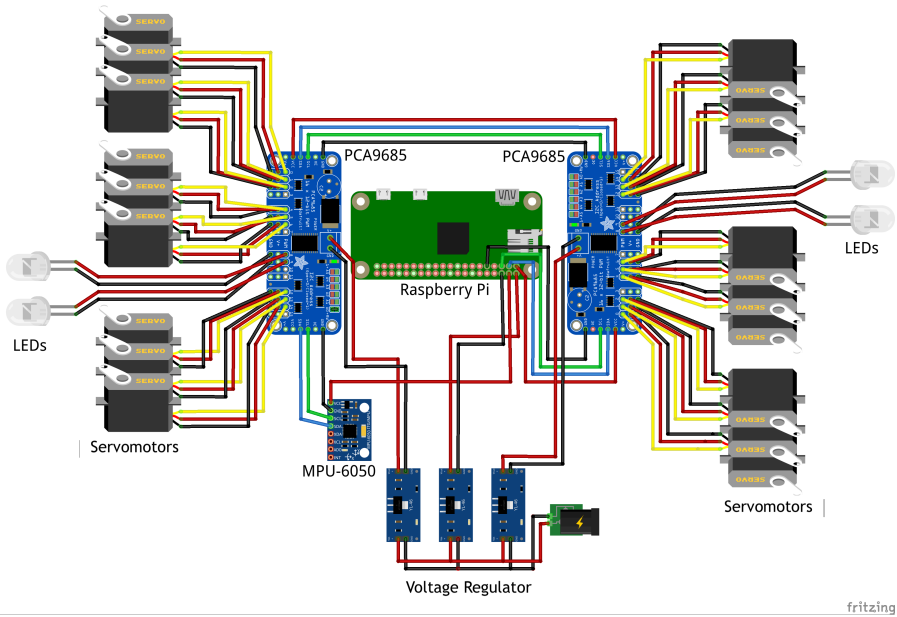

Figure 2. Robot's Electronic Architecture.

\subsection{Hardware}

The first challenge of this paper was to develop a low cost robot that could be easily reproduced. The other requirements were:

- 3 DoF (Degrees of Freedom) in each leg;

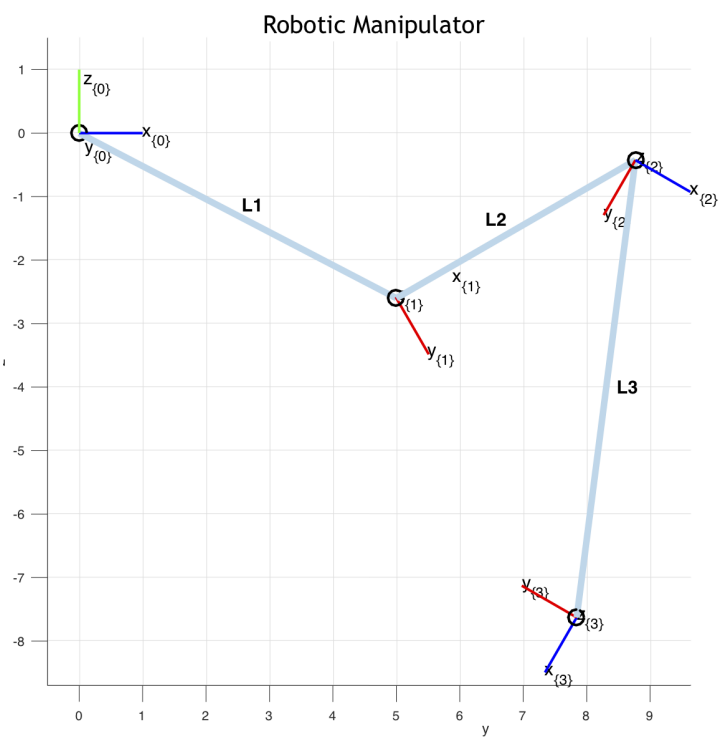

Figure 3. Modelled Leg on MATLAB ${ }^{\circledR}$

- A PWM (Pulse Width Modulation) Controller with at least 18 ports for the servomotors;

- A CPU (Central Processing Unit) with I2C (InterIntegrated Circuit) protocol for communication;

- A camera;

- Be able to communicate via WiFi;

- Battery should last at least 30 minutes.

A Raspberry Pi Zero W was used as main CPU. It is able to communicate via $\mathrm{I} 2 \mathrm{C}$ and $\mathrm{WiFi}$ protocols. A camera can be easily connected via CSI (Camera Serial Interface) connector. In order to control all the motors two PWM Controllers were used. Each one has 16 PWM outputs leaving 14 without use. Four LEDs were connected on some free ports, they are located in the bottom frame. Avoiding fragile plastic servomotors, metal servomotors were used. A 2-cell LiPo Battery were used to power up the entire system among with three voltage regulators. Looking for future works, a accelerometer and gyroscope module was installed in the project.

Fig. 2 represents the Robot's Electronic Architecture. The camera is connected to the Raspberry Pi CSI connector, not shown in Figure. This first prototype uses mainly developed modules. A main board will be developed for the second version of this hexapod.

The whole structure of the robot was made exclusively for it. Each leg was divided in three parts: Coxia, Femur and Tibia. They were made of PLA (Polylactic acid). The top frame was made in black piano acrylic and the bottom frame was made in transparent acrylic.

\subsection{Software}

A simple API (Application Programming Interface) was created so anyone can operate the robot. This API was developed in $\mathrm{C}++$ and contains basic functions specially developed for this hexapod. It is divided in two main libraries: Motion Control and Led Control.

The Motion Control is responsible for controlling all the movements and path planning of the robot. This library 


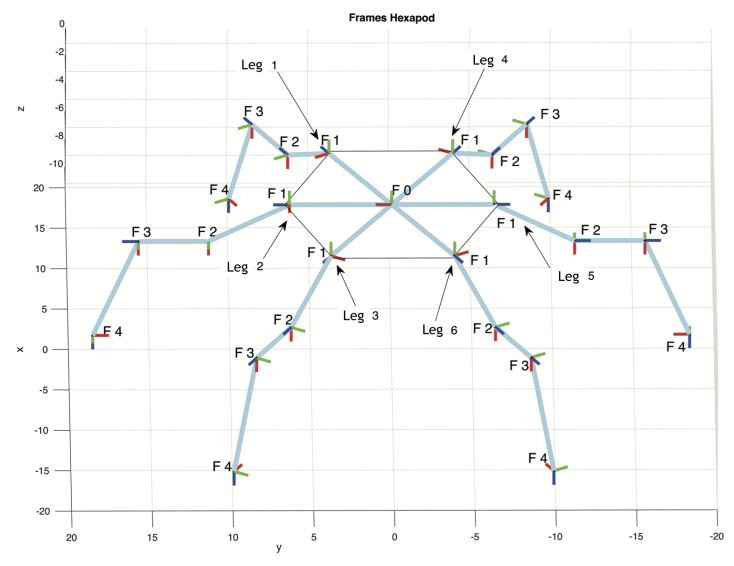

Figure 4. Modelled Robot on MATLAB ${ }^{\circledR}$

has functions that allow the final user to control from a single joint to the whole body at the same time. Inverse Kinematics and Trajectory Planning are also included in this library. It is possible to move the hexapod providing a speed in the coordinate axis $(\mathrm{x}, \mathrm{y})$. It is also possible to change the height of the robot.

The Led Control library was developed to control the four LEDs installed in the bottom frame of the robot. Calling a simple function from this library the user will be able to blink a single LED, change its intensity or perform an animation with all the LEDs.

\section{KINEMATICS}

In order to describe the whole Kinematics of an hexapod, each leg was modeled as a 3 DoF manipulator (Fig. 3). A model was built on MATLAB ${ }^{\circledR}$ so everything could be previously simulated.

Using Homogeneous Transformations it was possible to retrieve the Direct Kinematics of each leg. The hexapod configuration chosen was the circular/hexagonal one. This means that the robot is radially symmetric. All the legs are similar, they are disposed at $60^{\circ}$ from each other. Fig. 4 shows the complete model obtained.

The chosen method to obtain the Inverse Kinematics of each leg was the Jacobian (Pseudo-)Inverse Method. With this method is possible to retrieve the joint configurations using the differential Kinematics equation. The whole method can be represented by a control scheme presented in Fig. 5.

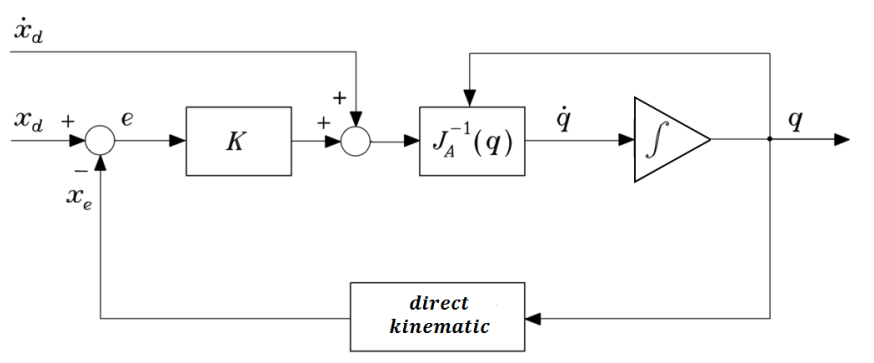

Figure 5. Jacobian (Pseudo-)Inverse Method adapted from Siciliano et al. (2010).

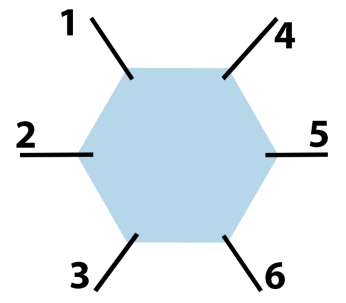

(a) Hexapod legs distribution

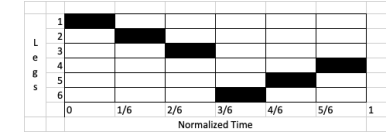

(d) Wave Gait
Figure 6. Gaits adapted from Oliveira (2016)

The use of the Inverse Kinematics makes possible to obtain the joint configurations from the cartesian's configurations. Unfortunately, only with the Inverse Kinematics solution to perform a motion can cause several damages to the robot. An example of this situation is during a movement its behaviour might not be smooth as planned and it can damage the actuators. In order to solve this problem a Trajectory Planning was implemented using a polynomial equation to interpolate the positions obtained via Inverse Kinematics. The method chosen was Point-toPoint motion with the Quintic Polynomial represented on Equation 1.

$$
\begin{gathered}
q_{i}(t)=a_{n-1} t^{n-1}+a_{n-2} t^{n-2}+\cdots+a_{2} t^{2}+a_{1} t+a_{0} \\
\theta\left(t_{0}\right)=\theta_{0}=a_{0}+a_{1} t_{0}+a_{2} t_{0}^{2}+a_{3} t_{0}^{3}+a_{4} t_{0}^{4}+a_{5} t_{0}^{5} \\
\theta\left(t_{f}\right)=\theta_{f}=a_{0}+a_{1} t_{f}+a_{2} t_{f}^{2}+a_{3} t_{f}^{3}+a_{4} t_{f}^{4}+a_{5} t_{f}^{5} \\
\dot{\theta}\left(t_{0}\right)=v_{0}=a_{1}+2 a_{2} t_{0}+3 a_{3} t_{0}^{2}+4 a_{4} t_{0}^{3}+5 a_{5} t_{0}^{4} \\
\dot{\theta}\left(t_{f}\right)=v_{f}=a_{1}+2 a_{2} t_{f}+3 a_{3} t_{f}^{2}+4 a_{4} t_{f}^{3}+5 a_{5} t_{f}^{4} \\
\ddot{\theta}\left(t_{0}\right)=\alpha_{0}=2 a_{2}+6 a_{3} t_{0}+12 a_{4} t_{0}^{2}+20 a_{5} t_{0}^{3} \\
\ddot{\theta}\left(t_{f}\right)=\alpha_{f}=2 a_{2}+6 a_{3} t_{f}+12 a_{4} t_{f}^{2}+20 a_{5} t_{f}^{3}
\end{gathered}
$$

The group of Equations 2 represents the Trajectory Planning for position $(\theta)$, speed $(v)$ and acceleration $(\alpha)$. The subscript 0 means initial and the subscript $f$ means final. The term $t_{0}$ means initial time and $t_{f}$ means final time.

With the Direct Kinematics, Inverse Kinematics and the Trajectory Planning implemented all the legs of the hexapod were able to achieve any position of its workspace.

\section{GAITS}

There are several different ways for a hexapod to walk. Three gaits are commonly observed in nature: wave gait, ripple gait, and tripod gait.

In Fig. 6 it is possible to observe the gaits mentioned before. The biggest difference between those is the amount of legs used to perform the movement. The white cells on Figure represents when each leg is touching the ground, 


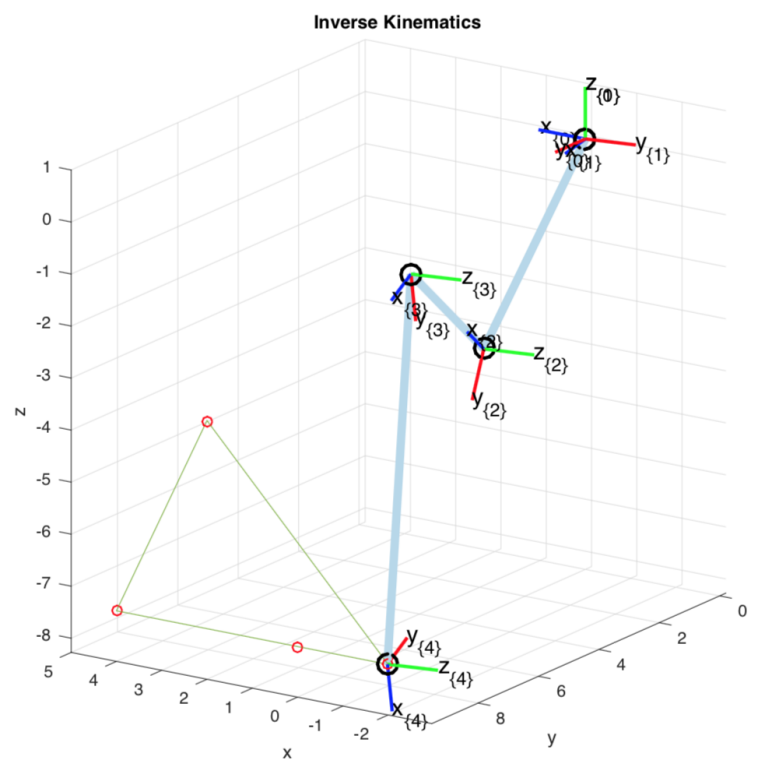

Figure 7. Tripod Gait using Inverse Kinematics

supporting the whole body. The black cells means that the leg is lifted up, moving to another position.

The tripod gait is the fastest among the others two gaits. The walking is divided in two steps: during the first step three of the legs stands on the floor supporting the whole body and the others are lifted, projecting themselves to the aimed direction. The second step is the opposite, the three legs who stayed as support now are lifted, projecting themselves to the aimed direction and the other three are supporting the robot.

Comparing to the others the wave gait is the slowest one. However, this gait is the most stable. It moves only a leg on each step.

Taking as an example, the tripod gait was chosen to be implemented on this hexapod.

\section{RESULTS}

In order to demonstrate the functionality of the Kinematics and Trajectory Planning it was proposed that one of the legs performs the Tripod Gait. The leg will lift up, project itself frontwards and than touch the ground before pulling the whole hexapod body. These steps were represented by 5 points in the Cartesian Coordinate Space, the first one is the original position of the leg and the other four are the gait positions. Figs. 7 and 8 show the movement made only with the Inverse Kinematics and the movement made with Trajectory Planning respectively. The green line represents the leg movement and the red circles the end-effector position. As noticed, the leg movement in the first case is not fluid as desired. However, the leg positioning was quite satisfactory. In the Trajectory Planning Method the variable time was used with 10 values between each previous point of the trajectory. Fig. 8 certifies that the movement became more fluid.

Figs. 9 and 10 show the variation of the angles Theta 1, Theta 2 and Theta 3 which corresponds to the Joint 1, Joint 2 and Joint 3 respectively for each experiment.

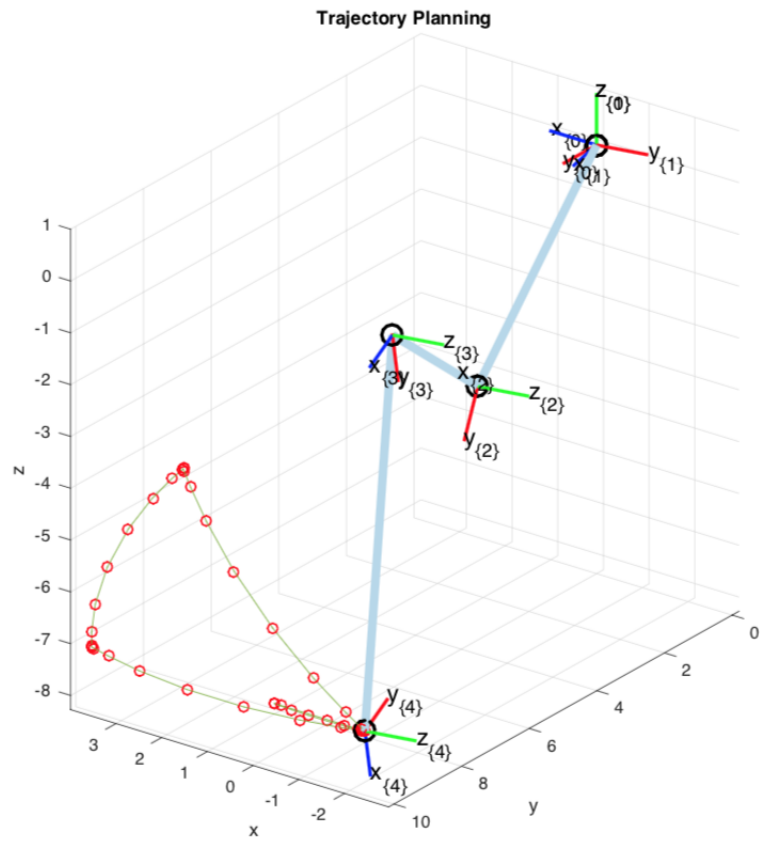

Figure 8. Tripod Gait using proposed Trajectory Planning Method
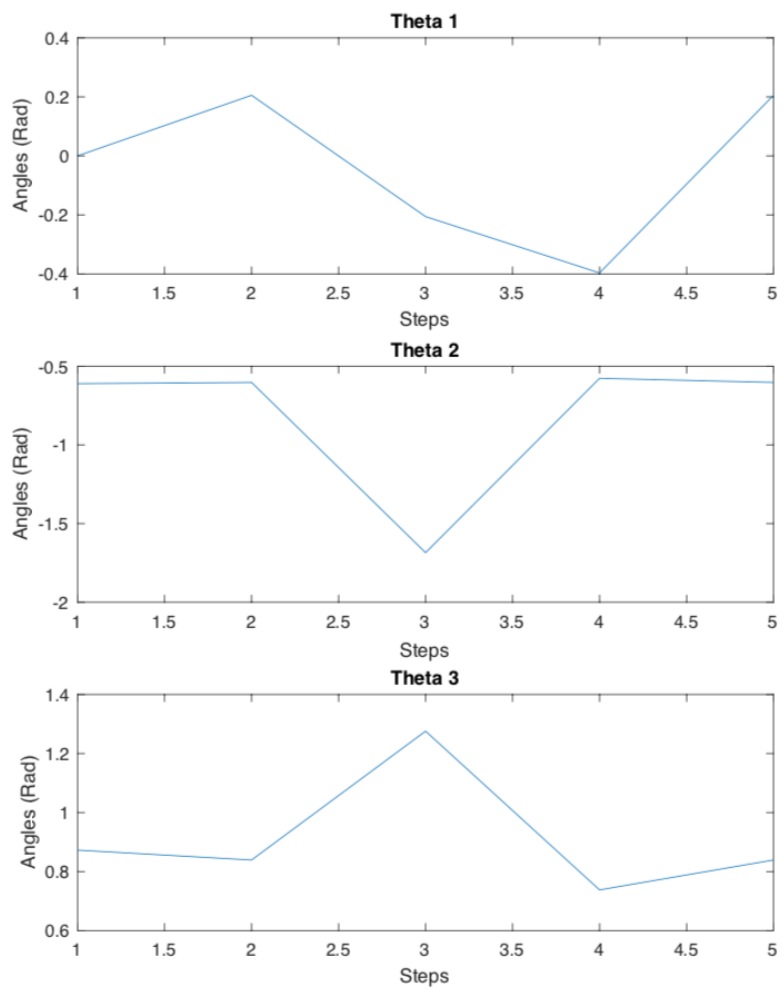

Figure 9. Joint angles variation using Inverse Kinematics

The same movement was executed on the real hexapod robot, reaffirming that this method works on both models simulated and real.

After attesting the efficiency of this method, the Tripod Gait was fully implemented on the real robot. The robot is holonomic and can walk among different heights. Is possible to see the programming simplicity and the robot 

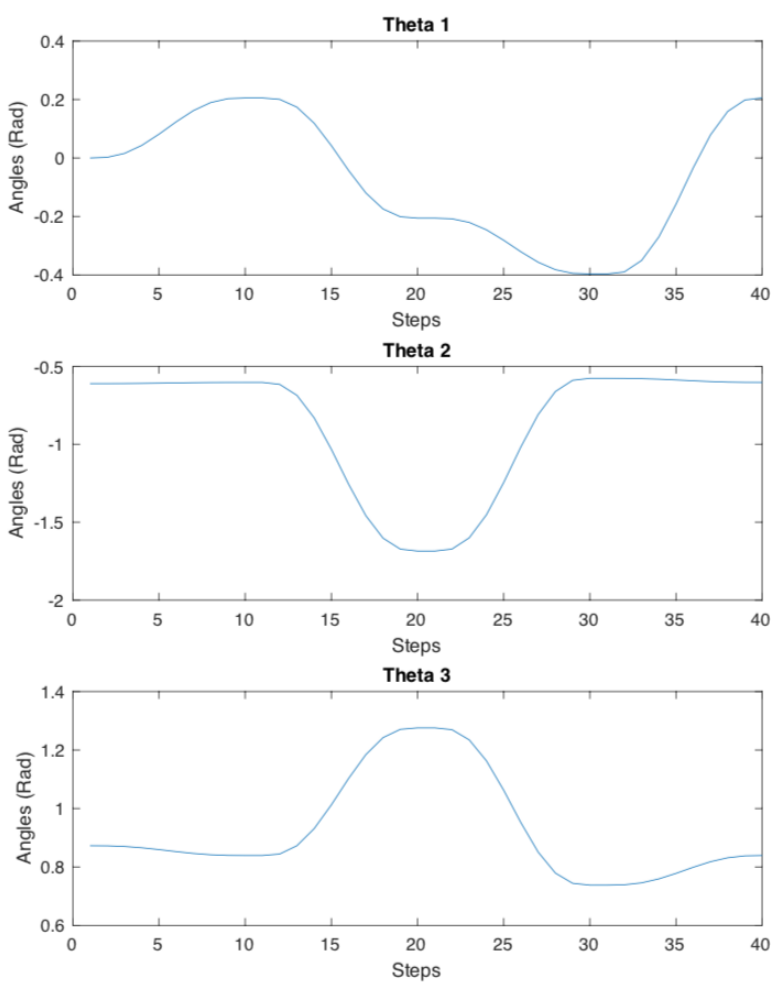

Figure 10. Joint angles variation using proposed Trajectory Planning Method

behavior in a demonstration video, available at https:// youtu.be/ikf0G7yx8c0.

The total price to build the robot was around $R \$ 750,00$ (Approximately $\$ 200,00)$. It is not a high value for a robot with a many servomotors, a camera and a Raspberry Pi.

\section{CONCLUSION}

This paper proposed the development and programming of a hexapod robot. The structural project was robust enough so the robot didn't have huge gaps between parts. The robot used cheap micro servomotors. However, they accomplished the task of lifting and carrying the robot.

The developed API makes easy to program the robot as simplicity is one of its concerns, thus not requiring a deep knowledge in algorithm which allows the robot operation by many student levels.

The hexapod's most common gaits were discussed. Even though Wave Gait is more stable, the Tripod Gait was chosen, once it is faster.

To control the hexapod's legs, (Pseudo-)Inverse Jacobian Method was proposed to execute their Inverse Kinematics, leading to a non-smooth movement. To cope with that, a quintic polynomial function was complementary used, and a smooth trajectory was acquired. Thus, the hexapod's gait become more fluid, and actuators effort decreased.

This platform is still under development. In future work, teaching material and manuals should be produced. A camera module still needs to be implemented. Motion functions also need to be improved. A integration with simulators and $\operatorname{ROS}^{\circledR}$ is also well seen for this project.

\section{REFERENCES}

Costelha, H. and Neves, C. (2018). Technical database on robotics-based educational platforms for k-12 students. In 2018 IEEE International Conference on Autonomous Robot Systems and Competitions (ICARSC), 167-172. doi:10.1109/ICARSC.2018.8374178.

Hudson, O.A., Fanni, M., Ahmed, S.M., and Sameh, A. (2018). Bio-inspired jumping maneuver for launching flapping wing micro air vehicles. In Autonomous Robot Systems and Competitions (ICARSC), 2018 IEEE International Conference on, 104-109. IEEE.

Kasaei, S.M., Lau, N., Pereira, A., and Shahri, E. (2017). A reliable model-based walking engine with push recovery capability. In Autonomous Robot Systems and Competitions (ICARSC), 2017 IEEE International Conference on, 122-127. IEEE.

Koh, J.S. and Cho, K.J. (2013). Omega-shaped inchworminspired crawling robot with large-index-and-pitch (lip) sma spring actuators. IEEE/ASME Transactions On Mechatronics, 18(2), 419-429.

Oliveira, L.F.P.d. (2016). Modelação, simulação e implementação de padrões de locomoção para robôs hexápodes. Ph.D. thesis.

Passault, G., Rouxel, Q., Petit, F., and Ly, O. (2016). Metabot: a low-cost legged robotics platform for education. In Autonomous Robot Systems and Competitions (ICARSC), 2016 International Conference on, 283-287. IEEE.

Siciliano, B., Sciavicco, L., Villani, L., and Oriolo, G. (2010). Robotics: modelling, planning and control. Springer Science \& Business Media.

Siegwart, R., Nourbakhsh, I.R., Scaramuzza, D., and Arkin, R.C. (2011). Introduction to autonomous mobile robots. MIT press.

Silveira, M.A. and Lacerda, W.S. (2017). Estudo, montagem e controle de um robô móvel hexápode. $X X X$ Congresso de Iniciação Científica da UFLA, Resumo. 